Article

\title{
Clinical Practice Use of Liquid Biopsy to Identify RAS/BRAF Mutations in Patients with Metastatic Colorectal Cancer (mCRC): A Single Institution Experience
}

\author{
Pietro Paolo Vitiello ${ }^{1,+}$, Vincenzo De Falco ${ }^{1,+} \mathbb{D}_{\text {, Emilio Francesco Giunta }}{ }^{1}$, Davide Ciardiello ${ }^{1}$, \\ Claudia Cardone ${ }^{1}$, Pasquale Vitale ${ }^{1}$, Nicoletta Zanaletti ${ }^{1}$, Carola Borrelli ${ }^{1}$, Luca Poliero ${ }^{1}$, \\ Marinella Terminiello ${ }^{1}$, Gianluca Arrichiello ${ }^{1}$, Vincenza Caputo ${ }^{1}$, Vincenzo Famiglietti ${ }^{1}$, \\ Valentina Mattera Iacono ${ }^{1}$, Francesca Marrone ${ }^{1}$, Alessandra Di Liello ${ }^{1}$, Giulia Martini ${ }^{1,2}$, \\ Stefania Napolitano ${ }^{1,3}{ }^{(D}$, Michele Caraglia ${ }^{4}\left(\mathbb{D}\right.$, Angela Lombardi ${ }^{4}$, Renato Franco ${ }^{5}$, \\ Ferdinando De Vita ${ }^{1}$, Floriana Morgillo ${ }^{1}$, Teresa Troiani ${ }^{1}$, Fortunato Ciardiello ${ }^{1}$ and \\ Erika Martinelli ${ }^{1, *}$
}

1 Department of Precision Medicine, Università della Campania "Luigi Vanvitelli", 80131 Napoli, Italy; pietropaolo.vitiello@gmail.com (P.P.V.); vincenzodefalc@gmail.com (V.D.F.); emiliofrancescogiunta@gmail.com (E.F.G.); davideciardiello@yahoo.it (D.C.); claudia.cardone88@gmail.com (C.C.); vitale.pasquale.89@gmail.com (P.V.); nicolettazanaletti@gmail.com (N.Z.); carola.bor@libero.it (C.B.); luca.poliero@libero.it (L.P.); marinella.terminiello@gmail.com (M.T.); gluca.arrichiello@gmail.com (G.A.); vincenza.caputo.92@gmail.com (V.C.); vincenzo.famiglietti@yahoo.it (V.F.); vmatteraiacono@gmail.com (V.M.I.); francesca.marrone@email.it (F.M.); dilielloalessandra@gmail.com (A.D.L.); giulia.mart14@gmail.com (G.M.); stenap@fastwebnet.it (S.N.); ferdinando.devita@unicampania.it (F.D.V.); floriana.morgillo@unicampania.it (F.M.); teresa.troiani@unicampania.it (T.T.); fortunato.ciardiello@unicampania.it (F.C.)

2 Centro Cellex, Vall D’Hebron Institute of Oncology (VHIO), 08035 Barcelona, Spain

3 Department of Gastrointestinal Medical Oncology, University of Texas, MD Anderson Cancer Center, Houston, TX 77030, USA

4 Department of Experimental Medicine, Università della Campania “Luigi Vanvitelli”, 80138 Napoli, Italy; michele.caraglia@unicampania.it (M.C.); angela.lombardi@unicampania.it (A.L.)

5 Department of Mental and Physical Health and Preventive Medicine, Pathology Unit, Università della Campania “Luigi Vanvitelli", 80138 Napoli, Italy; renato.franco@unicampania.it

* Correspondence: erika.martinelli@unicampania.it; Tel.: +39-081-5666628

+ These authors equally contributed to the work.

Received: 9 August 2019; Accepted: 2 October 2019; Published: 8 October 2019

\begin{abstract}
Tumor heterogeneity represents a possible cause of error in detecting predictive genetic alterations on tumor tissue and can be overcome by testing alterations in circulating tumor DNA (ctDNA) using liquid biopsy. We assessed 72 consecutive patients with a diagnosis of metastatic colorectal cancer (mCRC) using Idylla ${ }^{\mathrm{TM}}$ Biocartis, a fully automated platform that evaluates the most frequent mutations of KRAS, NRAS and BRAF genes. We correlated the results of liquid biopsy and standard tissue-based next generation sequencing (NGS) analyses to patient clinical features. The overall agreement was $81.94 \%$. Concordance was $85.71 \%$ and $96.15 \%$ in treatment-naïve patients and in the patient subgroup with liver metastases, respectively. In liver metastases positive, treatment-naïve patients, sensitivity, specificity and positive predictive value (PPV) were $92.31 \%$, $100 \%$ and $100 \%$, respectively. Circulating mutational fraction (CMF) was significantly higher in patients with liver metastases and high carcinoembryonic antigen (CEA) levels. In a subgroup of patients pre-treated with anti-Epidermal Growth Factor Receptor (EGFR) agents, emerging KRAS mutations were evidenced in $33 \%$ of cases. Testing $R A S / B R A F$ mutations on plasma using the Idylla ${ }^{\mathrm{TM}}$ Biocartis platform is feasible and reliable in $\mathrm{mCRC}$ patients in clinical practice.
\end{abstract}


Keywords: colorectal cancer; liquid biopsy; RAS testing; anti-EGFR; acquired resistance; clonal evolution

\section{Introduction}

Metastatic colorectal cancer (mCRC) is one of the main causes of cancer death worldwide, with 881,000 deaths in 2018 alone [1]. The addition of monoclonal antibodies (MoAbs) to chemotherapy has significantly prolonged survival in mCRC patients compared to chemotherapy alone [2]. Anti-Epidermal Growth Factor Receptor (EGFR) MoAbs, cetuximab and panitumumab, are currently administered in all-RAS (KRAS and NRAS) wild type (WT) mCRC patients as first or subsequent lines of treatment either in monotherapy or in association with chemotherapy, KRAS and NRAS tumor mutations being negative predictive biomarkers for their use. In fact, the presence of these mutations confers primary resistance to anti-EGFR in $\mathrm{mCRC}$ and it is mandatory to test for these mutations on tissue specimen before the initiation of anti-EGFR therapy [3]. Moreover, activating mutations of these genes could also develop during treatment with anti-EGFR in initially WT patients; this phenomenon is known as acquired (or secondary) resistance [4]. Liquid biopsy is an analytical technique consisting of the research of tumor-derived biomarkers in body fluids. Cell-free DNA (cfDNA) in the blood of mCRC patients includes, in different percentages, circulating tumor DNA (ctDNA) released by cancer cells, thus providing potential information in terms of prognosis and prediction of therapeutic sensitivity or resistance. Indeed, analysis of ctDNA has been evaluated in mCRC patients for different purposes: correlation between its levels and survival, monitoring of response to therapy, detection of $R A S / B R A F$ mutations at different time points [5]. In particular, the theoretical advantage of liquid biopsy over tissue biopsy in the metastatic and/or relapsed disease setting is the possibility to gain a full overview of the genetic make-up of the disease, overcoming both spatial and temporal heterogeneity [6]. In fact, various technologies are now available for testing known mutations in ctDNA in mCRC, the most used are based on digital PCR or quantitative PCR [7]. In particular, digital PCR shows the highest sensitivity, up to a limit of detection of $0.001 \%$ for digital droplet PCR [8-10] and about $0.1-1 \%$ for conventional quantitative PCR [11-13]. However, recent reports show how the highest sensitivity of the technique does not reflect a better prediction of the response to anti-EGFR agents, as it may include rare KRAS mutant subclones that do not have clinical significance [14-16]. The concordance between tissue and liquid biopsies in $\mathrm{mCRC}$, on the other hand, is strictly related not only to the technology used for tissue and plasma analyses, but also to some clinical parameters of the patients, such as the presence or absence of liver metastases [13,17-19]. Several retrospective and prospective studies have evaluated the use of liquid biopsy. The main purpose of these studies has generally been the evaluation of the degree of agreement between PCR on formalin-fixed paraffin-embedded (FFPE) tumor tissue and liquid biopsy, especially with beads, emulsion, amplification, magnetics (BEAMing) and digital droplet PCR (ddPCR) techniques [20-23]. In addition, some research focused on the use of liquid biopsy to guide treatments with anti-EGFR, evaluating the RAS status over time and the allelic frequency of mutations of this gene [24-27]. Similarly, a prospective study by the Franco-British Institute was performed using Idylla ${ }^{\mathrm{TM}}$ Biocartis on patients with $\mathrm{mCRC}$, showing an overall agreement with standard-of-care (SoC) of $73 \%$, which increased to $100 \%$ in patients with previously untreated metastatic liver disease [28]. Furthermore, in an analysis performed with the Idylla ${ }^{\mathrm{TM}}$ Biocartis platform in two first-line prospective clinical trials (PULSE, POSIBA), RAS and BRAF mutational status was assessed in ctDNA from 178 patients with KRAS exon 2 wild-type metastatic colorectal cancer with a sensitivity of $64.1 \%$ and a specificity of $90 \%$ [29]. Finally, preliminary data from the ongoing ERMES phase III trial in patients with KRAS/NRAS/BRAF WT mCRC also showed concordance of $83.8 \%$ of liquid biopsies with standard methods at baseline, with an increased frequency of mutant cases at progression disease (PD) after anti-EGFR treatment [30]. Another study, on the other hand, evaluated the possibility of using anti-EGFR drugs in subsequent lines of therapy, in patients who had RAS mutation on the primary tumor and were $R A S$ WT on the Idylla ${ }^{\mathrm{TM}}$ platform, after treatments with 
anti-angiogenic drugs [31]. Here we describe our experience with liquid biopsy testing for KRAS, NRAS and BRAF mutations in $\mathrm{mCRC}$, using Idylla ${ }^{\mathrm{TM}}$ Biocartis, a fully automated real-time PCR-based platform, in 72 consecutive patients from two groups: patients who had not received prior medical treatment for mCRC (basal cohort) and patients who had received anti-EGFR agents for the treatment of mCRC (post-EGFR cohort).

\section{Results}

\subsection{Concordance between Tissue and Plasma Samples}

A total of 72 patients were analyzed by the Idylla ${ }^{\mathrm{TM}}$ Biocartis platform: 47 patients were all-RAS and BRAF WT, 22 patients were KRAS mutated (14 on codon 12, 2 on codon 13, 2 on codon 61, 1 on codon 117, 3 on codon 146), 1 patient was NRAS mutated (on codon 61), 2 were BRAF mutated (on codon 600). The distribution of clinically relevant characteristics in the study population are shown in Supplementary Table S1. The overall concordance between tissue analysis and liquid biopsy was $81.94 \%$ (59/72; kappa 0.590; 95\% CI: 0.392-0.789). Concordance was also calculated according to different patient subgroups: patients without liver metastases (NON-LIVER) and patients with at least one liver metastasis (LIVER POSITIVE), further divided into patients with metastases limited to the liver (LIVER ONLY) and patients with metastases in the liver and other organs (LIVER and OTHER) (Table 1A). Considering only the liquid biopsies performed on the $42 \mathrm{mCRC}$ patients that were evaluated before starting first line therapy (BASAL COHORT), the concordance raised to $85.71 \%(36 / 42$; kappa 0.714 ; $95 \%$ CI: $0.507-0.922)$ with a maximum concordance of $96.15 \%(25 / 26$; kappa $0.923 ; 95 \%$ CI: $0.776-1$ ) in patients with at least one liver metastasis (LIVER POSITIVE) and 100\% in patients with metastases not limited to the liver (LIVER and OTHER) (Table 1B). For more details on the discordant cases in the basal cohort, refer to Table 2.

Table 1. Concordance, sensitivity, specificity and positive predictive value according to patients' cohort. (A) GLOBAL COHORT; (B) BASAL COHORT. NON-LIVER: patients with no evidence of liver metastases; LIVER ONLY: patients with metastases limited to the liver; LIVER and OTHER: patients with metastases not limited to the liver; LIVER POSITIVE: all the patients with liver metastases (the sum of LIVER ONLY and LIVER and OTHER); TP: true positive; TN: true negative; FP: false positive; FN: false negative; PPV: positive predictive value.

\begin{tabular}{|c|c|c|c|c|c|c|c|c|c|}
\hline $\mathbf{A}$ & $\mathbf{N}^{\circ}$ & TP & TN & FP & FN & Concordance $\%$ & Sensitivity \% & Specificity \% & PPV \% \\
\hline Global cohort & 72 & 17 & 42 & 8 & 5 & 81.94 & 77.27 & 84 & 68 \\
\hline Non-liver & 23 & 4 & 13 & 2 & 4 & 73.91 & 50 & 86.67 & 66.67 \\
\hline Liver positive & 49 & 12 & 30 & 6 & 1 & 85.71 & 92.31 & 83.33 & 66.67 \\
\hline Liver only & 13 & 2 & 8 & 2 & 1 & 76.92 & 66.67 & 80 & 50 \\
\hline Liver and other & 36 & 10 & 22 & 4 & 0 & 88.89 & 100 & 84.62 & 71.43 \\
\hline B & $\mathbf{N}^{\circ}$ & TP & TN & FP & FN & Concordance $\%$ & Sensitivity \% & Specificity \% & PPV \% \\
\hline Basal cohort & 42 & 16 & 20 & 1 & 5 & 85.71 & 76.19 & 95.24 & 94.12 \\
\hline Non-liver & 16 & 4 & 7 & 1 & 4 & 68.75 & 50 & 87.5 & 80 \\
\hline Liver positive & 26 & 12 & 13 & 0 & 1 & 96.15 & 92.31 & 100 & 100 \\
\hline Basal liver only & 8 & 3 & 4 & 0 & 1 & 87.5 & 75 & 100 & 100 \\
\hline Basal liver and other & 18 & 9 & 9 & 0 & 0 & 100 & 100 & 100 & 100 \\
\hline
\end{tabular}


Table 2. Discordant cases in the basal cohort (see text for details). MAF: mutation allele fraction; WT: wild type.

\begin{tabular}{|c|c|c|c|c|}
\hline Case & Results of Tissue & Results of Liquid Biopsy & Clinical Characteristics & Possible Explanations \\
\hline A & KRAS MUT (G12C) & KRAS WT, NRAS WT, BRAF WT & $\begin{array}{l}\text { Pelvic-infiltrating, inoperable rectal } \\
\text { cancer. No distant metastasis. }\end{array}$ & $\begin{array}{l}\text { The absence of distant metastases is associated with low } \\
\text { abundance of circulating tumor DNA (ctDNA). }\end{array}$ \\
\hline $\mathrm{B}$ & KRAS MUT (G13D) & KRAS WT, NRAS WT, BRAF WT & $\begin{array}{l}\text { Left colon cancer with } \\
\text { loco-regional disease. }\end{array}$ & $\begin{array}{l}\text { The absence of distant metastases is associated with low } \\
\text { abundance of circulating tumor DNA (ctDNA). }\end{array}$ \\
\hline $\mathrm{C}$ & KRAS MUT (G12V) & KRAS WT, NRAS WT, BRAF WT & $\begin{array}{l}\text { Left colon cancer with subcentimetric } \\
\text { nodal disease. }\end{array}$ & $\begin{array}{l}\text { The absence of distant metastases is associated with low } \\
\text { abundance of circulating tumor DNA (ctDNA). }\end{array}$ \\
\hline $\mathrm{D}$ & NRAS MUT (Q61H) & KRAS WT, NRAS WT, BRAF WT & $\begin{array}{l}\text { Left colon cancer with multiple } \\
\text { subcentimetric liver metastases. }\end{array}$ & $\begin{array}{l}\text { Low MAF for this mutation on ctDNA (predicted sensitivity } \\
\text { is optimal for MAF }>5 \% \text { ). The liquid biopsy performed at } \\
\text { disease progression (tumor burden increased) confirmed } \\
\text { NRAS mutation. }\end{array}$ \\
\hline $\mathrm{E}$ & $B R A F$ MUT (V600E) & KRAS WT, NRAS WT, BRAF WT & $\begin{array}{l}\text { Right colon cancer with multiple } \\
\text { centimetric lung metastases. }\end{array}$ & $\begin{array}{l}\text { Patient with non-liver metastatic disease and a low burden } \\
\text { of disease. Low MAF for this mutation on ctDNA } \\
\text { (predicted sensitivity is optimal for MAF }>5 \% \text { ). }\end{array}$ \\
\hline $\mathrm{F}$ & $\begin{array}{l}\text { KRAS WT, NRAS } \\
\text { WT, BRAF WT }\end{array}$ & NRAS MUT (G13D) & $\begin{array}{l}\text { Nodal-limited recurrence of } \\
\text { left colon cancer. }\end{array}$ & $\begin{array}{l}\text { The relapsed cancer is enriched with NRAS mutant cells, } \\
\text { that were missed on tissue analysis. }\end{array}$ \\
\hline
\end{tabular}




\subsection{Sensitivity, Specificity and Positive Predictive Value (PPV)}

Overall, sensitivity, specificity and PPV were $77.27 \%, 84 \%$ and $68 \%$, respectively. Analyses of these parameters for different subgroups defined according to patients' metastatic sites are also shown in Table 1A. The highest sensitivity $(100 \%)$ was reached in the LIVER and OTHER subgroup with a specificity of $84.62 \%$ and a PPV of $71.43 \%$. Sensitivity, specificity and PPV were also calculated in the cohort of treatment-naive patients (BASAL COHORT), considering the subgroups previously described (Table 1B).

Finally, receiver operating characteristic (ROC) curves for the basal cohort were obtained. The area under the curve (AUC) for the basal patient group was 0.8571 (CI: 0.7335-0.9808), whereas the AUC for the liver metastases positive patient subgroup was 0.9615 (CI: 0.8746-1.000) (Figure 1).

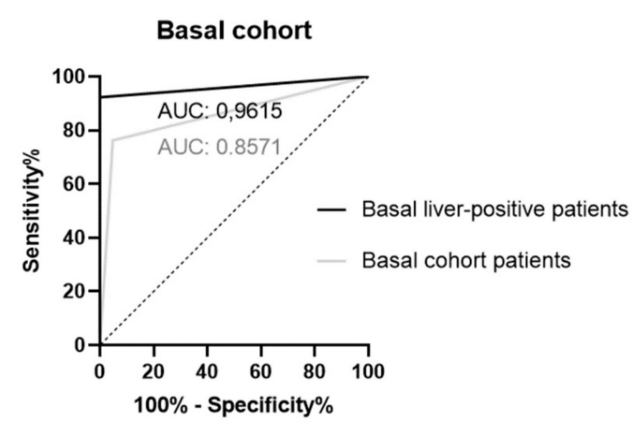

Figure 1. Receiver operating characteristic (ROC) curves for the BASAL COHORT patients (light grey, AUC: 0.8571, CI: 0.7335-0.9808) and for the subgroup of basal LIVER POSITIVE patients (black, AUC: 0.9615, CI: 0.8746-1.000).

\subsection{Circulating Mutational Fraction}

The circulating mutational fraction $(\mathrm{CMF})$ was calculated for all the tests that were positive for a $R A S$ mutation, representing the relative amount of RAS mutated DNA over total cfDNA, as described in the Materials and Methods section. For this reason, we hypothesized that CMF values should be higher for those patients with higher tumor DNA shedding and thus with higher levels of ctDNA over cfDNA. For this purpose, we analyzed the distribution of the CMF values in the $42 \mathrm{mCRC}$ basal cohort patients according to sites of metastases and to CEA level, that were used as a surrogate of tumor burden, as previously described [32]. The median CMF values for the non-liver and for the liver metastases positive subgroup were $0.36 \%$ (range $0.0002-10.88 \%$ ) and $8.24 \%$ (range $2.54-23.8 \%$ ), respectively, with a statistically significant difference between the two subgroups (Figure 2A). In addition, an exploratory analysis evidenced a statistically significant difference between the distribution of the CMF values according to CEA levels above or below the median of the values ( $46.5 \mathrm{ng} / \mathrm{mL}$ ) (Figure 2B).

A

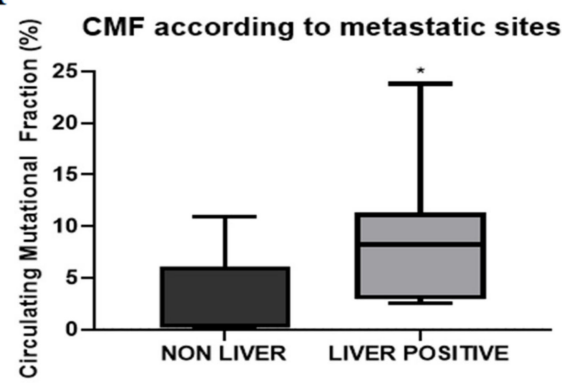

B

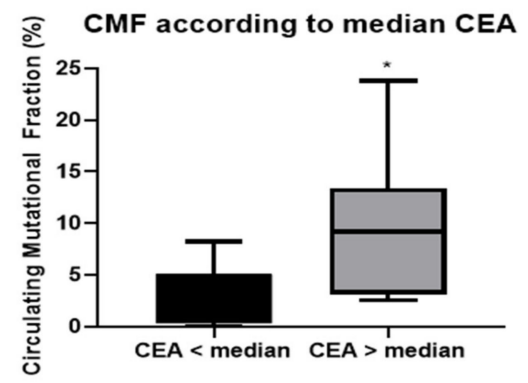

Figure 2. Circulating mutational fraction (CMF) distribution between clinical subgroups. (A) Difference between NON-LIVER (5 pts) and LIVER POSITIVE (9 pts) metastatic colorectal cancer (mCRC) $(p=0.0385)$. (B) Difference between patients with baseline CEA levels below (7 pts) or above (7 pts) median $(p=0.0469) .{ }^{*}: p<0.05$. NON-LIVER: patients with no evidence of liver metastases. LIVER POSITIVE: all the patients with at least one liver metastasis (the sum of LIVER ONLY and LIVER and OTHER). 


\subsection{Post-Anti-EGFR Cohort}

This cohort consisted of $30 \mathrm{mCRC}$ patients with RAS wild-type tumors on tissue analysis that had received systemic anti-EGFR therapy during treatment for metastatic disease. Liquid biopsy identified 10 KRAS mutated patients in this group: 4 codon 61 mutations, 2 codon 146 mutations, 3 codon 12 mutations and 1 case of double positivity for codon 12 and codon 61 mutations. Notably, the distribution of these mutations differs from that found in the $42 \mathrm{mCRC}$ patients that were evaluated before first line treatment, with a higher prevalence of exon 3 and exon 4 as compared to exon 2 mutations, as previously reported [4].

Further, 15 out of these 30 patients were treated with an anti-EGFR drug as a re-challenge treatment strategy. For these patients, serial liquid biopsies were performed during re-challenge therapy. KRAS mutations were found during the course of treatment in 5 out of $15(33.3 \%)$ patients. We further assessed the CMF variation of KRAS mutations during anti-EGFR re-challenge treatment, comparing the value at the first evidence of KRAS mutant circulating clones (T1) and at a following analysis at 6-8 weeks (T2). The continuation of anti-EGFR therapy leads to a substantial and significant increase of CMF in these patients (Figure 3).

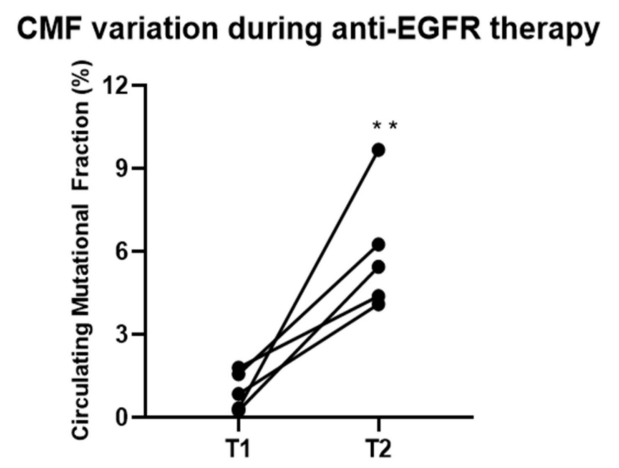

Figure 3. $\mathrm{CMF}$ variation during anti-Epidermal Growth Factor Receptor (EGFR) re-challenge treatment $(p=0.0079)$. T1: first evidence of KRAS mutant circulating clones, T2: analysis of KRAS circulating mutations $6-8$ weeks after T1. ${ }^{* *}: p<0.01$.

\section{Discussion}

The present study has evaluated in a single institution in a clinical practice setting the level of concordance, sensitivity, specificity and PPV of Idylla ${ }^{\mathrm{TM}}$ qPCR-based platform by Biocartis on plasma samples in comparison to next generation sequencing (NGS) analysis on tumor specimens in mCRC patients. We have found that these parameters vary according to metastatic sites and according to previous treatments. Concerning the concordance of results, these data are in line with previous reports that have been performed with the Idylla ${ }^{\mathrm{TM}}$ Biocartis system [29-31], with the concordance higher in mCRC patients for which the analysis has been performed at baseline of metastatic disease $(85.71 \%)$, especially for those patients with liver metastases $(96.15 \%)$, who also showed the highest sensitivity $(100 \%)$. Collectively these data suggest that, in patients with these characteristics, RAS/BRAF status could effectively be identified by the Idylla ${ }^{\mathrm{TM}}$ Biocartis liquid biopsy-based platform. However, the specificity was different between different groups of patients: $84 \%$ and $95.24 \%$, respectively in the global and basal cohorts. Collectively, these data suggest that it is possible to identify mutations in the plasma and, therefore, more accurately define the correct mutation status despite a $R A S / B R A F$ wild-type result on tissue analysis in approximately 1 out of 6 patients and 1 out of 21 patients, respectively in the global and in the basal cohort. Finally, a PPV of $94.12 \%$ with the liquid biopsy analysis in the correct detection of mutations in mCRC before first line treatment suggests that this technique is a good alternative to NGS-based testing in clinical practice, especially for those cases in which it is not easy to obtain evaluable tumor tissue. Indeed, the reliability of the test coupled to the very short turn-around time of only about $2 \mathrm{~h}(85-130 \mathrm{~min})$ from plasma collection to complete 
mutational report make this technique worthy for this clinical setting. Furthermore, the detection of mutations in the plasma during the course of anti-EGFR-based therapies reflects the emergence and/or the selection of RAS mutant clones under the pressure of anti-EGFR MoAbs and, therefore, could potentially allow a dynamic monitoring of disease evolution and efficacy of therapies. For this reason, the analyses for concordance, sensitivity and specificity compared to primary tumor tissue are not useful in the post-EGFR cohort of patients. Notably, in this cohort of patients we found a single case of double positivity (KRAS codon 12 and 61). It is known that tumor heterogeneity could lead to the development of several mutated clones, but the clinical relevance of them is still debated. In the work by Thierry et al. [33], a higher percentage of samples harbored more than one mutation, but this reflects the higher sensitivity of IntPlexVR used for ctDNA analysis; in particular, the lower the threshold, the higher the percentage of more-than-one mutation detected. Idylla ${ }^{\mathrm{TM}}$ has a lower sensitivity but this could probably eliminate a "background noise" of clinically irrelevant mutations.

Finally, to our knowledge, this study provides the first evidence that by using the Idylla ${ }^{\mathrm{TM}}$ Biocartis platform for circulating tumor DNA it is possible to calculate the CMF for those cases positive for RAS or BRAF mutation. This value represents the fraction of mutant DNA over total cfDNA and, as such, it highly depends on the quantity of non-tumor circulating DNA. Nonetheless, CMF is directly correlated with both the abundance of total ctDNA and the mutation allele fraction (MAF). Although further prospective data are needed in this context, CMF might be used as a practical clinical surrogate of these two parameters.

In the 42 basal mCRC patient cohort, this value significantly correlates with the presence of liver metastases and with higher CEA levels, the latter being used as a surrogate for tumor burden. Further, even with the limitation of a small sample size (a total of five patients), the CMF of acquired KRAS mutations increases over time in patients treated with anti-EGFR agents as re-challenge therapy. In the frame of a serial testing for RAS mutations in liquid biopsies in patients undergoing anti-EGFR therapy, a raise in CMF levels might represent a potential marker of cancer cell resistance onset and might anticipate the clinical evidence of disease progression and, thus, might allow a rational change in cancer therapy.

\section{Learning from the Discordant Cases}

Among the discordant cases in the $42 \mathrm{mCRC}$ patient basal cohort (Table 2), we have identified three cases (patients A-C) with a KRAS mutation in NGS tissue analysis and RAS WT status in liquid biopsy. These three patients did not have liver metastases, a condition that is more often associated with less ctDNA shedding in the blood. In fact, as confirmed in other works using even more sensitive techniques, the absence of liver metastases was the also main clinical factor associated with inconclusive circulating tumor DNA results in previous reports [19].

A fourth case of RAS mutation identified in NGS tissue analysis but not in liquid biopsy analysis (patient D) refers to a patient with low tumor burden (multiple subcentimetric liver metastases, not amenable to resection). Furthermore, since this was a NRAS mutation, the sensitivity of the Idylla ${ }^{\mathrm{TM}}$ Biocartis platform to identify NRAS mutations is not high enough for such a case, since it requires at least $5 \%$ of allelic frequency for optimal detection of these mutations [34]. Interestingly, when a subsequent plasma analysis was performed at disease progression, the same NRAS mutation previously evidenced in NGS tissue testing at baseline (Q61H) was detected, suggesting cancer cell clonal expansion. A BRAF V600E mutation was detected in NGS tissue analysis but not in liquid biopsy in a case (patient E) with low tumor burden and lung limited metastatic disease. Similarly to NRAS mutations, the optimal detection for BRAF V600 mutations using Idylla ${ }^{\mathrm{TM}}$ Biocartis platform for plasma is above $5 \%$ of mutated allele frequency [34]. Interestingly, in one case (patient F), NRAS mutation was detected in liquid biopsy at diagnosis of tumor recurrence, while the NGS analysis of primary tumor tissue resulted in RAS wild type. This patient was treated with anti-EGFR agents in first line as the standard of care but experienced a disease progression at the first evaluation after only 3 months of therapy, as generally occurs with primary resistance. This case underlines the potential 
advantage of performing liquid biopsy at the time of tumor recurrence as compared to tissue analysis of the primary tumor for a better clinical prediction on the efficacy of anti-EGFR therapies.

\section{Materials and Methods}

\subsection{Study Oversight}

Patients provided informed consent for an institutional review board-approved protocol for longitudinal collection of plasma and profiling on tumor DNA within the I-Cure research program. Between 1 July 2018 and 30 June 2019, 72 patients were evaluable for analysis. Among them, 42 patients were assessed at baseline (i.e., before the initiation of any systemic treatment for $\mathrm{mCRC}$ ): basal cohort; whereas 30 patients with all KRAS/NRAS WT on tissue analysis were treated with a therapy regimen including an anti-EGFR MoAb (cetuximab or panitumumab) and analyzed for liquid biopsy during the course of treatment for metastatic disease: post-EGFR cohort.

\subsection{Plasma Collection}

At least $6 \mathrm{~mL}$ of whole blood was collected by standard procedure peripheral vein blood draw, using Vacutainer ${ }^{\circledR}$ with EDTA as anticoagulant (K2EDTA, purple cap, catalog \#367863, Becton Dickinson). Plasma was separated through two different centrifugation steps (the first at room temperature for $10 \mathrm{~min}$ at $1500 \times g$ and the second at $2000 \times g$ for the same time and temperature). Plasma was stored at $-80{ }^{\circ} \mathrm{C}$ until analysis.

\subsection{Mutational Analyses of Tissue Specimens}

Analyses of tissue specimens were all performed in the Pathology Service of Università della Campania "Luigi Vanvitelli" using Next Generation Sequencing (NGS).

\subsubsection{Sample Preparation}

An appropriate formalin-fixed paraffin-embedded (FFPE) tissue block was selected for each case. Four unstained FFPE tissue sections were cut at $10 \mu \mathrm{m}$ each for DNA extraction. DNA was obtained using the QIAamp ${ }^{\circledR}$ DNA FFPE kit Tissue (Qiagen, Duesseldorf, Germany), according to the manufacturer's instructions. Extracted DNA was eluted in 20 or $30 \mu \mathrm{L}$ of elution buffer and then DNA was quantified by a Qubit ${ }^{\circledR}$ 2.0 Fluorometer (Life Technologies, Singapore) using the Qubit ${ }^{\circledR}$ dsDNA HS Assay kit, according to the manufacturer's recommendations. The extracted DNA was stored at $-20{ }^{\circ} \mathrm{C}$. The Ion Torrent Personal Genome Machine (PGM) technology allows the massive parallel sequencing of DNA libraries, of several different samples, using an approach based on the $\mathrm{PH}$ variations that occur at the moment of incorporation of the single deoxyribonucleotide into the reaction catalyzed by the DNA polymerase.

\subsubsection{Library Preparation}

Around $10 \mathrm{ng}$ of DNA was used to prepare the sequencing libraries. The libraries were prepared with the IonAmpliSeq ${ }^{\mathrm{TM}}$ Library kit 2.0 (Thermo Fisher Scientific, Carlsbad, CA, USA) and with primer pool: IonAmpliSeq Colon and Lung Cancer Research Panel v2, which analyzes 504 mutational hotspots and targeted regions in 22 genes, including KRAS, BRAF and NRAS.

Amplified products were purified with Agencourt AMPure XP beads (Beckman Coulter Genomics, High Wycombe, UK). Concentrations of amplified and bar-coded libraries were measured using the Qubit ${ }^{\circledR} 2.0$ Fluorometer (Life Technologies, Singapore) and the Qubit ${ }^{\circledR}$ dsDNA HS Assay kit (Life Technologies, Singapore). DNA libraries were stored at $-20{ }^{\circ} \mathrm{C}$. The libraries were clonally amplified on Ion Sphere ${ }^{\mathrm{TM}}$ particles after dilution of the libraries to $100 \mathrm{pM}$. Template preparation was performed with the IonOneTouch ${ }^{\mathrm{TM}} 2$ System (Thermo Fisher Scientific, Carlsbad, CA, USA), an automated system for emulsion PCR, recovery of Ion Sphere ${ }^{\mathrm{TM}}$ particles and enrichment of template-positive particles. The Ion Sphere ${ }^{\mathrm{TM}}$ particles coated with template were applied to the semiconductor chip. A short 
centrifugation step was conducted to allow the spherical particles to be deposited into the chip wells. Finally, sequencing was carried out using Ion $316^{\mathrm{TM}}$ chips on the Ion Personal Genome Machine System $\left(\mathrm{PGM}^{\mathrm{TM}}\right.$, Thermo Fisher Scientific) using the Ion $\mathrm{PGM}^{\mathrm{TM}} \mathrm{Hi}-\mathrm{Q}$ view Sequencing kit v2 (Thermo Fisher Scientific, Carlsbad, CA, USA).

\subsubsection{Data Analysis}

The Torrent Suite Software v.4.0.2 (Life Technologies) was used to assess run performance and data analysis. Integrative Genomics Viewer (IGV v 2.2, Broad Institute) was used for visual inspection of the aligned reads. Sequencing data were analyzed using Ion Reporter software (https: //ionreporter.lifetechnologies.com/) and further filtered through quality checking. We selected all single nucleotide variants (SNVs) in the studied genes resulting in a non-synonymous amino acid change, or a premature stop codon, and all short indels resulting in either a frameshift or insertion/ deletion of amino acids. All SNVs were analyzed for previously reported hotspot mutations (somatic mutations reported in COSMIC database(cancer.sanger.ac.uk) and novel variations, i.e., new mutations detected by NGS but not reported in either COSMIC or db SNP databases.

\subsection{Mutational Analyses of Plasma}

Analyses of plasma were all carried out using the automated Idylla ${ }^{\mathrm{TM}}$ qPCR-based platform by Biocartis. Briefly, $1 \mathrm{~mL}$ of fresh plasma was added to the specific cartridge for automated analysis. The whole procedure takes less than $1 \mathrm{~min}$ hands-on time and about $2 \mathrm{~h}$ for the analysis ( 85 to $130 \mathrm{~min}$ ). ctKRAS and ctNRAS/BRAF/EGFR cartridges were used. The mutations detected by the cartridges are: ctKRAS: exon 2 (G12 > C/R/S/A/D/V, G13 > D), exon $3(\mathrm{~A} 59>\mathrm{E} / \mathrm{G} / \mathrm{T}, \mathrm{Q} 61>\mathrm{K} / \mathrm{L} / \mathrm{R} / \mathrm{H})$, exon $4(\mathrm{~K} 117>\mathrm{N}$, A146 > P/T/V); ctNRAS/BRAF/EGFR: NRAS exon 2 (G12 > C/S/A/D/V, G13 > D/V/R), exon 3 (A59 > T, $\mathrm{Q} 61>\mathrm{K} / \mathrm{L} / \mathrm{R} / \mathrm{H})$, exon $4(\mathrm{~K} 117>\mathrm{N}, \mathrm{A} 146>\mathrm{T} / \mathrm{V})$, BRAF V600 > E/D/K/R, EGFR S492 > R. The limit of detection of the Idylla ${ }^{\mathrm{TM}}$ platform for cfDNA is set at about 10,000 copies of WT DNA (about $30 \mathrm{ng}$ ) per milliliter. At the end of the run the platform displays an automated report that includes: validity of the run (in case no DNA is detected, the test is considered invalid), presence or absence of mutation(s), type of mutation(s) detected, Cq of the total KRAS/NRAS/BRAF, Cq of the specific mutation(s) detected. No specific training is required in order to interpret the test result.

In order to evaluate the reproducibility of this test in our series, we collected plasma samples of the first 10 patients in duplicate at a short time interval $(6-48 \mathrm{~h})$. Mutation analysis with Idylla ${ }^{\mathrm{TM}}$ platform was performed twice for this set of samples: if the result had been discordant, the analysis would have been repeated for the third time. Considering 100\% concordance between two consecutive samples in the first set of 10 patients, no additional analysis was performed for the following cases. Additionally, in the case of discordance between plasma and tissue-based or previous plasma-based analyses, a confirmatory plasma sample was acquired and re-analyzed.

\subsection{Concordance}

Accuracy was calculated using the interrate agreement kappa (K) coefficient [35]. The strength of agreement was interpreted according to $\mathrm{K}$ value as follows: poor $(<0.20)$, fair $(0.21-0.40)$, moderate (0.41-0.60), good (0.61-0.80) and very good (0.81-1.00). All analyses were performed using Graphpad online Kappa calculator.

\subsection{Sensitivity, Specificity and Positive Predictive Value}

Assuming the NGS analysis of FFPE as gold standard analysis, the results of the liquid biopsy analysis were defined as true positive (TP) when the same mutation was found in both NGS and liquid biopsy analyses, true negative (TN) when no mutation was detected by both analyses, false positive (FP) when a specific mutation was only found in liquid biopsy analysis and false negative (FN) when a specific mutation was detected only by NGS of FFPE. Sensitivity was defined as the ability of the liquid biopsy platform to detect mutations in patients with known alterations in RAS/BRAF genes by NGS 
analysis of FFPE (sensitivity $=\mathrm{TP} /(\mathrm{TP}+\mathrm{FN})$ ). Specificity was defined as the proportion of patients without mutations in both tissue and plasma among patients without known genetic alterations by NGS analysis of FFPE (specificity $=\mathrm{TN} /(\mathrm{TN}+\mathrm{FP})$ ). Positive predictive value (PPV) was defined as the probability that a mutation detected in the plasma sample was also present in the tissue sample $(\mathrm{PPV}=\mathrm{TP} /(\mathrm{TP}+\mathrm{FP}))$.

\subsection{Circulating Mutational Fraction}

$\Delta \mathrm{Cq}$ values, defined as the difference between the $\mathrm{Cq}$ of the mutation and the mean $\mathrm{Cq}$ of the internal control, were obtained for each test that was positive for a mutation. The percentage of mutated DNA over the internal control was calculated as follows: $2-\Delta \mathrm{Cq} \times 100$; this value was defined as the circulating mutational fraction (CMF). In the basal cohort, median CMF values were compared between the liver metastases positive patient group and the liver metastases negative group, using the Mann-Whitney U test. Median CMF values were also compared according to CEA levels, by using the same test. In the subgroup of patients undergoing anti-EGFR re-challenge therapy, the CMF values of the acquired $R A S$ mutations were compared between the first evidence and the re-analysis at 6-8 weeks, by using the Wilcoxon rank sum test. All statistical analyses were performed using Graphpad (Prism 8).

\section{Conclusions}

We have shown how using a simple PCR-based assay for $R A S$ and $R A F$ testing in plasma of mCRC patients is convenient and clinically relevant. Taking into account the reliability and the repeatability of the described method, as well as the short turn-around time and the sustainable costs [36], this approach could be feasible and effective in the clinical practice management of mCRC patients.

Supplementary Materials: The following are available online at http://www.mdpi.com/2072-6694/11/10/1504/s1, Table S1: Patients' clinical characteristics across the three cohorts used in the analyses.

Author Contributions: Conceptualization, P.P.V., F.C. and E.M.; Data curation, P.P.V., V.D.F. and E.F.G.; Formal analysis, P.P.V., V.D.F. and E.F.G.; Funding acquisition, F.C. and E.M.; Investigation, P.P.V., V.D.F. and E.F.G.; Methodology, P.P.V., V.D.F., E.F.G., D.C. and C.C.; Resources, V.F., V.M.I., F.M. (Francesca Marrone) and A.D.L.; Supervision, T.T., F.C. and E.M.; Validation, G.M., S.N., M.C., A.L., R.F. and F.D.V.; Visualization, P.V., N.Z., C.B., L.P., M.T., G.A., V.C., G.M. and S.N.; Writing—original draft, P.P.V., V.D.F. and E.F.G.; Writing—review and editing, P.V., N.Z., R.F., F.D.V., F.M. (Floriana Morgillo), T.T., F.C. and E.M.

Funding: This research was funded by Associazione Italiana per la Ricerca sul Cancro (AIRC) to F.C. and Regione Campania Cancer Research Campaign I-Cure Grant to F.C.

Acknowledgments: The Authors wish to thank all the patients who participated to the study.

Conflicts of Interest: P.P.V.: consultancy for Biocartis. E.M.: advisory board for Amgen, Bayer, Merck, Roche, Sanofi, Servier and expert opinion for ESMO (European Society of Medical Oncology). T.T.: advisory board for Amgen, Bayer, Merck, Novartis, Roche, Sanofi. F.M.: advisory board for Lilly, MSD. F.D.V.: advisory board for Amgen, Lilly, Roche, Celgene. F.C.: advisory board for Merck, Roche, Amgen, Bayer, Servier, Symphogen, Pfizer and research funding from Roche, Merck, Amgen, Bayer, Ipsen. V.D.F., E.F.G., D.C., C.C., C.B., L.P., M.T., G.A., V.C., A.D.L., V.F., V.M.I., F.M., G.M., S.N., R.F., A.L., M.C. declare no competing conflict of interest regarding the following manuscript. The funders had no role in the design of the study; in the collection, analyses, or interpretation of data; in the writing of the manuscript, or in the decision to publish the results.

\section{References}

1. Latest Global Cancer Data: Cancer Burden Rises to 18.1 Million New Cases and 9.6 Million Cancer Deaths in 2018; WHO: Geneva, Switzerland, 2018; Volume 3.

2. Tol, J.; Punt, C.J. Monoclonal antibodies in the treatment of metastatic colorectal cancer: A review. Clin. Ther. 2010, 32, 437-453. [CrossRef] [PubMed]

3. Van Cutsem, E.; Cervantes, A.; Adam, R.; Sobrero, A.; van Krieken, J.H.; Aderka, D.; Aguilar, E.A.; Bardelli, A.; Benson, A.; Bodokyet, G.; et al. ESMO consensus guidelines for the management of patients with metastatic colorectal cancer. Ann. Oncol. 2016, 27, 1386-1422. [CrossRef] [PubMed] 
4. Siravegna, G.; Mussolin, B.; Buscarino, M.; Corti, G.; Cassingena, A.; Crisafulli, G.; Ponzetti, A.; Cremolini, C.; Amatu, A.; Lauricella, C.; et al. Clonal evolution and resistance to EGFR blockade in the blood of colorectal cancer patients. Nat. Med. 2015, 21, 795-801. [CrossRef] [PubMed]

5. Normanno, N.; Cervantes, A.; Ciardiello, F.; De Luca, A.; Pinto, C. The liquid biopsy in the management of colorectal cancer patients: Current applications and future scenarios. Cancer Treat. Rev. 2018, 70, 1-8. [CrossRef] [PubMed]

6. Dagogo-Jack, I.; Shaw, A.T. Tumor heterogeneity and resistance to cancer therapies. Nat. Rev. Clin. Oncol. 2018, 15, 81-94. [CrossRef]

7. Siravegna, G.; Marsoni, S.; Siena, S.; Bardelli, A. Integrating liquid biopsies into the management of cancer. Nat. Rev. Clin. Oncol. 2017, 14, 531-548. [CrossRef]

8. Diehl, F.; Li, M.; He, Y.; Kinzler, K.W.; Vogelstein, B.; Dressman, D. BEAMing: Single-molecule PCR on microparticles in water-in-oil emulsions. Nat. Methods 2006, 3, 551-559. [CrossRef]

9. Sanmamed, M.F.; Fernández-Landázuri, S.; Rodríguez, C.; Ruth, Z.; María, D.L.; Leyre, Z.; José Luis, P.; Salvador, M.; Alvaro, G. Quantitative cell-free circulating BRAFV600E mutation analysis by use of droplet digital PCR in the follow-up of patients with melanoma being treated with BRAF inhibitors. Clin. Chem. 2015, 61, 297-304. [CrossRef]

10. Hindson, B.J.; Ness, K.D.; Masquelier, D.A.; Belgrader, P.; Heredia, N.J.; Makarewicz, A.J.; Bright, I.J.; Lucero, M.Y.; Hiddessen, A.L.; Legler, T.C.; et al. High-Throughput Droplet Digital PCR System for Absolute Quantitation of DNA Copy Number. Anal. Chem. 2011, 83, 8604-8610. [CrossRef]

11. Oh, J.E.; Lim, H.S.; An, C.H.; Jeong, E.G.; Han, J.Y.; Lee, S.H.; Yoo, N.J. Detection of Low-Level KRAS Mutations Using PNA-Mediated Asymmetric PCR Clamping and Melting Curve Analysis with Unlabeled Probes. J. Mol. Diagn. 2010, 12, 418-424. [CrossRef]

12. Watanabe, K.; Fukuhara, T.; Tsukita, Y.; Morita, M.; Suzuki, A.; Tanaka, N.; Terasaki, H.; Nukiwa, T.; Maemondo, M. EGFR Mutation Analysis of Circulating Tumor DNA Using an Improved PNA-LNA PCR Clamp Method. Can. Respir. J. 2016, 2016, 5297329. [CrossRef] [PubMed]

13. Vivancos, A.; Aranda, E.; Benavides, M.; Élez, E.; Gómez-España, M.A.; Toledano, M.; Alvarez, M.; Parrado, M.R.C.; García-Barberán, V.; Diaz-Rubio, E. Comparison of the Clinical Sensitivity of the Idylla Platform and the OncoBEAM RAS CRC Assay for KRAS Mutation Detection in Liquid Biopsy Samples. Sci. Rep. 2019, 9, 8976. [CrossRef] [PubMed]

14. Normanno, N.; Esposito Abate, R.; Lambiase, M.; Forgione, L.; Cardone, C.; Iannaccone, A.; Sacco, A.; Rachiglio, A.M.; Martinelli, E.; Rizzi, D.; et al. RAS testing of liquid biopsy correlates with the outcome of metastatic colorectal cancer patients treated with first-line FOLFIRI plus cetuximab in the CAPRI-GOIM trial. Ann. Oncol. 2018, 29, 112-118. [CrossRef] [PubMed]

15. Laurent-Puig, P.; Pekin, D.; Normand, C.; Steve, K.K.; Philippe, N.; Karla, P.; Rachel, R.; Jeff, O.; Preethi, S.; Delphine, 1.; et al. Clinical relevance of KRAS-mutated subclones detected with picodroplet digital PCR in advanced colorectal cancer treated with anti-EGFR therapy. Clin. Cancer Res. 2015, 21, 1087-1097. [CrossRef] [PubMed]

16. Vidal, J.; Bellosillo, B.; Vivas, C.S.; García-Alfonso, P.; Carrato, A.; Cano, M.; García-Carbonero, R.; Élez, E.; Losa, F.; Massutí, B.; et al. Ultra-selection of metastatic colorectal cancer patients using next-generation sequencing to improve clinical efficacy of anti-EGFR therapy. Ann. Oncol. 2019, 30, 439-446. [CrossRef] [PubMed]

17. Grasselli, J.; Élez, E.; Caratú, G.; Matito, J.; Santos, C.; Macarulla, T.; Vidal, J.; García, M.; Viéitez, J.M.; Páez, D.; et al. Concordance of blood- and tumor-based detection of RAS mutations to guide anti-EGFR therapy in metastatic colorectal cancer. Ann. Oncol. 2017, 28, 1294-1301. [CrossRef] [PubMed]

18. Elez, E.; Chianese, C.; Sanz-García, E.; Martinelli, E.; Noguerido, A.; Mancuso, F.M.; Caratù, G.; Matito, J.; Grasselli, J.; Cardone, C.; et al. Impact of circulating tumor DNA mutant allele fraction on prognosis in RAS -mutant metastatic colorectal cancer. Mol. Oncol. 2019, 13, 1827-1835. [CrossRef]

19. Bachet, J.B.; Bouché, O.; Taieb, J.; Dubreuil, O.; Garcia, M.L.; Meurisse, A.; Normand, C.; Gornet, J.M.; Artru, P.; Louafi, S.; et al. RAS mutation analysis in circulating tumor DNA from patients with metastatic colorectal cancer: The AGEO RASANC prospective multicenter study. Ann. Oncol. 2018, 29, 1211-1219. [CrossRef]

20. Taly, V.; Pekin, D.; Benhaïm, L.; Kotsopoulos, S.K.; Le Corre, D.; Li, X.; Atochin, I.; Link, D.R.; Griffiths, A.D.; Pallier, K.; et al. Multiplex Picodroplet Digital PCR to Detect KRAS Mutations in Circulating DNA from the Plasma of Colorectal Cancer Patients. Clin. Chem. 2013, 59, 1722-1731. [CrossRef] 
21. Bettegowda, C.; Sausen, M.; Leary, R.J.; Kinde, I.; Wang, Y.; Agrawal, N.; Bartlett, B.R.; Wang, H.; Luber, B.; Alani, R.M.; et al. Detection of circulating tumor DNA in early- and late-stage human malignancies. Sci. Transl. Med. 2014, 6, 224ra24. [CrossRef]

22. Thierry, A.R.; Mouliere, F.; El Messaoudi, S.; Mollevi, C.; Lopez-Crapez, E.; Rolet, F.; Gillet, B.; Gongora, C.; Déchelotte, P.; Robert, B.; et al. Clinical validation of the detection of KRAS and BRAF mutations from circulating tumor DNA. Nat. Med. 2014, 20, 430-435. [CrossRef] [PubMed]

23. Wan, J.C.M.; Massie, C.; Garcia-Corbacho, J.; Mouliere, F.; Brenton, J.D.; Caldas, C.; Pacey, S.; Baird, R.; Rosenfeld, N. Liquid biopsies come of age: Towards implementation of circulating tumour DNA. Nat. Rev. Cancer 2017, 17, 223-238. [CrossRef] [PubMed]

24. Takayama, Y.; Suzuki, K.; Muto, Y.; Ichida, K.; Fukui, T.; Kakizawa, N.; Ishikawa, H.; Watanabe, F.; Hasegawa, F.; Saito, M.; et al. Monitoring circulating tumor DNA revealed dynamic changes in KRAS status in patients with metastatic colorectal cancer. Oncotarget 2018, 9, 24398-24413. [CrossRef] [PubMed]

25. García-Foncillas, J.; Tabernero, J.; Élez, E.; Aranda, E.; Benavides, M.; Camps, C.; Jantus-Lewintre, E.; López, R.; Muinelo-Romay, L.; Montagut, C.; et al. Prospective multicenter real-world RAS mutation comparison between OncoBEAM-based liquid biopsy and tissue analysis in metastatic colorectal cancer. Br. J. Cancer 2018, 119, 1464-1470. [CrossRef] [PubMed]

26. Toledo, R.A.; Cubillo, A.; Vega, E.; Garralda, E.; Alvarez, R.; de la Varga, L.U.; Pascual, J.R.; Sánchez, G.; Sarno, F.; Prieto, S.H.; et al. Clinical validation of prospective liquid biopsy monitoring in patients with wild-type RAS metastatic colorectal cancer treated with FOLFIRI-cetuximab. Oncotarget 2017, 8, 35289-35300. [CrossRef]

27. Spindler, K.-L.G.; Pallisgaard, N.; Appelt, A.L.; Andersen, R.F.; Schou, J.V.; Nielsen, D.; Pfeiffer, P.; Yilmaz, M.; Johansen, J.S.; Hoegdall, E.V.; et al. Clinical utility of KRAS status in circulating plasma DNA compared to archival tumour tissue from patients with metastatic colorectal cancer treated with anti-epidermal growth factor receptor therapy. Eur. J. Cancer 2015, 51, 2678-2685. [CrossRef] [PubMed]

28. Chibaudel, B. Extended RAS Mutational Status Analysis in Circulating Tumor DNA from Patients with Advanced Colorectal Cancer in Daily Clinical Practice. The Franco-British Institute Experience and Recommendations. Biomed. J. Sci. Tech. Res. 2018, 6. [CrossRef]

29. Maurel, J.; Alonso, V.; Escudero, P.; Fernández-Martos, C.; Salud, A.; Méndez, M.V.; Gallego, V.; Rodriguez, J.R.; Martín-Richard, M.; Fernández-Plana, J.; et al. Clinical Impact of Circulating Tumor RAS and BRAF Mutation Dynamics in Patients with Metastatic Colorectal Cancer Treated with First-Line Chemotherapy Plus Anti-Epidermal Growth Factor Receptor Therapy. JCO Precis. Oncol. 2019, 1-16. [CrossRef]

30. Normanno, N.; Barone, C.; Maiello, E.; Di Costanzo, F.; Cassata, A.; Tamburini, E.; Tonini, G.; Bordonaro, R.; Rosati, G.; Zaniboni, A.; et al. Analysis of liquid biopsies from metastatic colorectal carcinoma (mCRC) patients (pts) enrolled in the ERMES clinical trial. J. Clin. Oncol. 2018, 36, e15507. [CrossRef]

31. Raimondi, C.; Nicolazzo, C.; Belardinilli, F.; Loreni, F.; Gradilone, A.; Mahdavian, Y.; Gelibter, A.; Giannini, G.; Cortesi, E.; Gazzaniga, P. Transient Disappearance of RAS Mutant Clones in Plasma: A Counterintuitive Clinical Use of EGFR Inhibitors in RAS Mutant Metastatic Colorectal Cancer. Cancers 2019, 11, 42. [CrossRef]

32. Berger, A.W.; Schwerdel, D.; Welz, H.; Marienfeld, R.; Schmidt, S.A.; Kleger, A.; Ettrich, T.J.; Seufferlein, T. Treatment monitoring in metastatic colorectal cancer patients by quantification and KRAS genotyping of circulating cell-free DNA. PLoS ONE 2017, 12, e0174308. [CrossRef] [PubMed]

33. Thierry, A.R.; El Messaoudi, S.; Mollevi, C.; Raoul, J.L.; Guimbaud, R.; Pezet, D.; Artru, P.; Assenat, E.; Borg, C.; Mathonnet, M.; et al. Clinical utility of circulating DNA analysis for rapid detection of actionable mutations to select metastatic colorectal patients for anti-EGFR treatment. Ann. Oncol. 2017, 28, 2149-2159. [CrossRef] [PubMed]

34. ctNRAS-BRAF cartridge Technical Sheet. Available online: https://media.biocartis.com/biocartis/documents/ Tech_Sheet-ctNRAS-BRAF-IVD-A4_WEB.pdf (accessed on 29 June 2019).

35. Landis, J.R.; Koch, G.G. The measurement of observer agreement for categorical data. Biometrics 1977, 33, 159-174. [CrossRef] [PubMed]

36. Bellosillo, B.; Pages, J.; Collin, C.; Pasmans, R.; Montagut, C. The Cost of Molecular Diagnostic Testing in Oncology-A Workflow Analysis. Value Heal. 2015, 18, A336-A337. [CrossRef]

(C) 2019 by the authors. Licensee MDPI, Basel, Switzerland. This article is an open access article distributed under the terms and conditions of the Creative Commons Attribution (CC BY) license (http://creativecommons.org/licenses/by/4.0/). 